\title{
sciforum
}

\section{CONICET A Mass Spectrometry-based Lipidomics Study for Early Diagnosis of clear cell Renal Cell Carcinoma}

C I B I O N

Malena Manzi ${ }^{1}$, Martín Palazzo ${ }^{2,3}$, Nicolás Zabaleguii ${ }^{1}$, María Elena Knottt ${ }^{1}$, Patricio Yankilevich ${ }^{3}$, María Isabel Giménez ${ }^{4}$, Lyida I. Puricelli5, María Eugenia Monge*1

Roffo ${ }^{1}$ Centro de Investigaciones en Bionanociencias (CIBION), Consejo Nacional de Investigaciones Científicas y Técnicas (CONICET), Godoy Cruz 2390, C1425FQD, CABA, Argentina. ${ }^{2}$ Instituto de Investigación en Biomedicina de Buenos Aires (IBioBA) CONICET, Instituto Partner de la Sociedad Max Planck, Godoy Cruz 2390, C1425FQD, CABA, Argentina. ${ }^{3}$ LM2S, Université de Technologié de Troyes 12 rue Marie-Curie, CS42060 Troyes, France. ${ }^{4}$ Departamento de Diagnóstico y Tratamiento, Hospital Italiano de Buenos Aires, Tte. Gral. Juan Domingo Perón 4190

C1199ABB, CABA, Argentina. Instituto de Oncología Ángel H. Roffo, Facultad de Medicina, Universidad de Buenos Aires, Av. San Martín 5481, C1417DTB, CABA, Argentina. HOSPITAL ITALIANO *maria.monge@cibion.conicet.gov.ar

INTRODUCTION
Kidney cancer is fundamentally a metabolic disease ${ }^{1}$ and more than $30 \%$ of patients, often incidentally
diagnosed by imaging procedures, exhibit locally advanced or metastatic renal cell carcinoma (RCC) at the time
of diagnosis. ${ }^{2,3}$ The disease is inherently resistant to chemotherapy ${ }^{4}$ and radiotherapy. ${ }^{5}$ Clear cell $R C C$ (ccRCC) is
the most common (75\%) lethal subtype, and is considered a glycolytic and lipogenic tumor. ${ }^{6,7}$ Current research
has shown that several metabolic alterations are associated with RCC and different potential biomarkers have
been identified. ${ }^{7-9}$ Early diagnosis is needed to reduce the mortality associated to ccRCC, to give more
opportunities for early intervention and improved outcome of ccRCC patients. In this context, lipids are
candidate molecules to be explored in a non-targeted fashion as potential biomarkers for ccRCC diagnosis by
means of lipid profiling experiments.

Patient Cohort - Retrospective Study

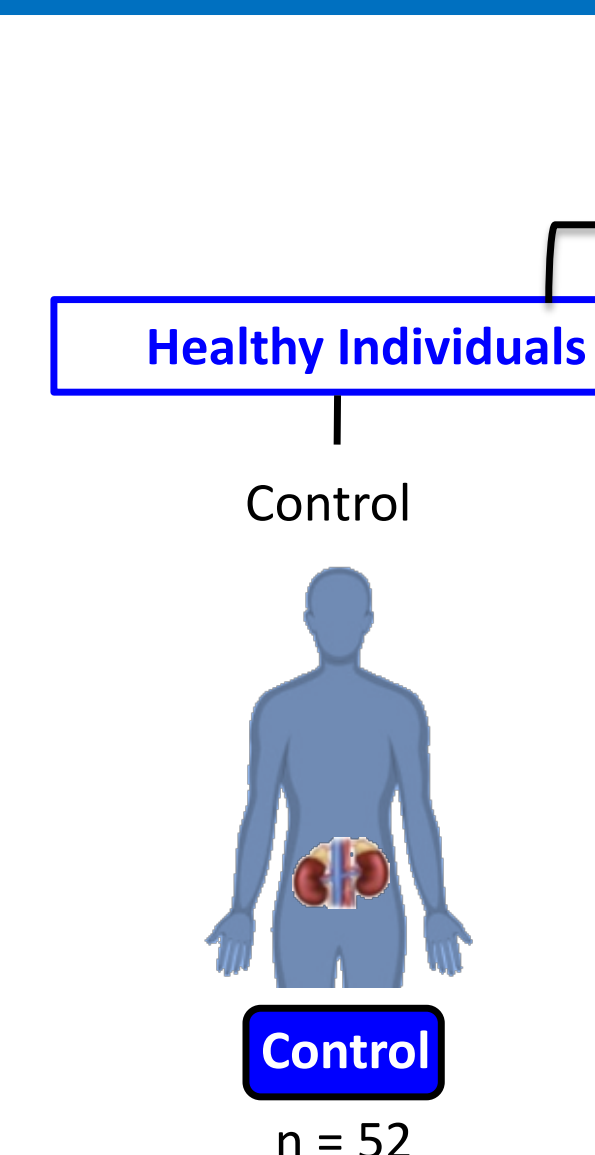
Human Serum Samples (n=112)

\begin{tabular}{c} 
The analysis was performed on age-matched serum samples \\
\hline \hline BPMS(1)
\end{tabular}

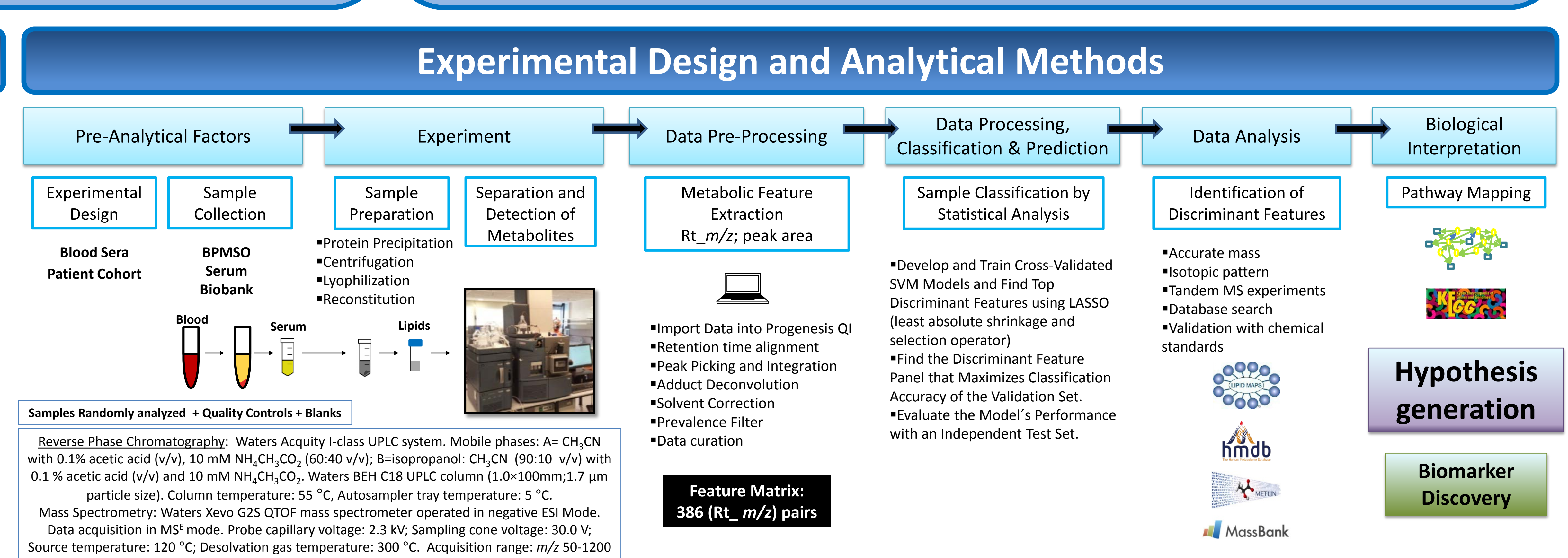

RESULTS

Experimental Design and Analytical Methods

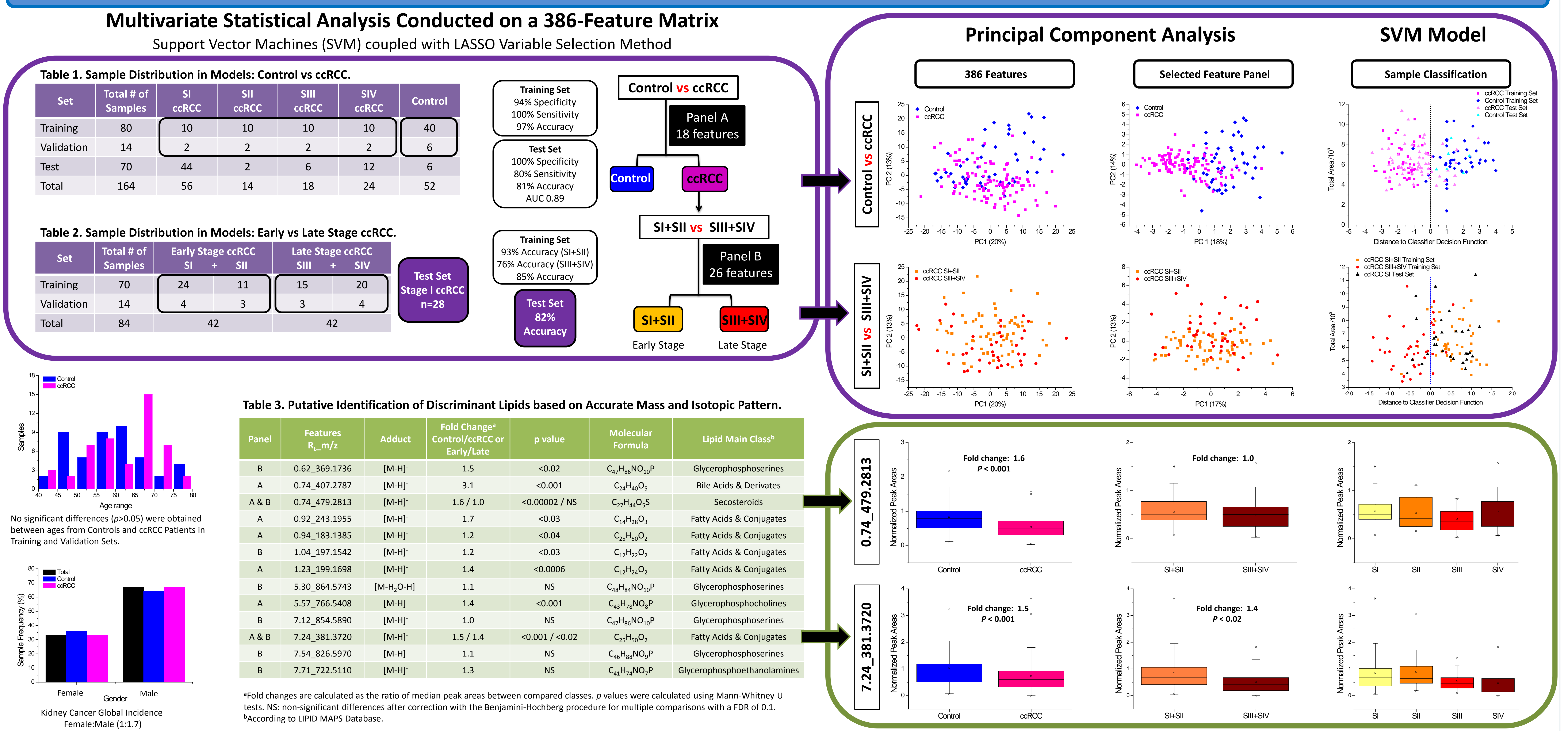

\section{Conclusions \& Perspectives}

- Lipid profiling coupled with SVM-LASSO multivariate analysis provided 2 discriminant feature panels for serum sample classification and prediction: i) 18 features allowed discriminating controls from ccRCC patients with $81 \%$ accuracy in an independent test set, and ii) 26 features allowed classifying stage I from stage III and IV ccRCC patients in an independent test set with $82 \%$

\section{Acknowledgment \\ We acknowledge CONICET, ANPCYT, and MINCYT for providing the funding, and the BPMSO Biobank from}

controls, in agreement with previous studies.

- 2 discriminant lipids are common to both panels, 42 lipids would allow early ccRCC detection. Current work involves the identification of the discriminant lipids by tandem MS experiments and comparison with chemical standards.

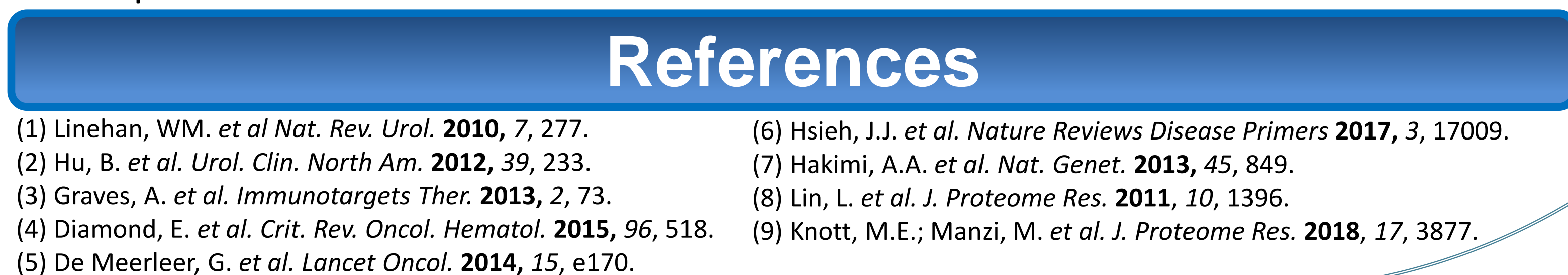

- Profile the lipidome using a discovery-based lipidomics approach via UHPLC-OTOF-MS.

Compare the serum lipid profiles of ccRCC patients with those from healthy individuals.

Compare the lipid profiles along disease progression through the analysis of samples from patients with

different ccRCC stages $(I, I I, I I I, I V)$.

Develop a machine learning method applying Support Vector Machines (SVM) with LASSO to find An

ide re 